Paediatr Paedolog 2018 $53: 141-143$ https://doi.org/10.1007/s00608-018-0603-4

(c) Springer-Verlag GmbH Austria, ein Teil von Springer Nature 2018

CrossMark

\title{
Klaus Schmitt
}

Klinik für Kinder- und Jugendheilkunde, Kepler Universitätsklinikum, Linz, Österreich

\section{Von der Primärversorgung zum Whole Genome Sequencing}

Zunehmend können freie KassenstelDas Thema der 56. Jahrestagung der Österreichischen Gesellschaft für Kinderund Jugendheilkunde (ÖGKJ), die vom 27. bis 29. September 2018 in Linz stattfindet, wurde bewusst gewählt, um wieder einmal auf die Breite unseres Fachs aufmerksam zu machen. Von öffentlichen Institutionen wird die Pädiatrie oft anders gesehen. Dabei sind wir wahrscheinlich in der Ausbildung das letzte große Fach mit allen Subspezialitäten.

\section{》) Die ÖGKJ befindet sich} heute im Spannungsfeld - von Primärversorgung bis Whole Genome Sequencing

Neben einer notwendigen Organmedizin unter Rücksichtnahme auf die physischen und psychischen Entwicklungsstadien der Kinder und Jugendlichen kommen noch sozialmedizinische Aspekte der Familien dazu, die zu beachten sind. Dieser ganzheitliche Aspekt ist für unsere Patienten ein großer Vorteil und gleichzeitig das Befriedigende unserer täglichen Arbeit.

\section{Pädiater sind Primärversorger}

Wir sind Primärversorger, werden aber nicht immer so wahrgenommen. Bei der Diskussion um Primary-HealthCare-Zentren wird die Pädiatrie in manchen Bundesländern übergangen. Bei der Oberösterreichischen Gebietskrankenkasse wurde ich wiederholt mit der Aussage konfrontiert: „Das können auch Allgemeinmediziner!“ Dabei wird vergessen, dass Allgemeinmediziner in ihrer Ausbildung maximal 3 Monate Pädiatrie absolvieren. len nicht nachbesetzt werden. Welche Gründe sind dafür maßgeblich? Das Arbeitszeitgesetz hat die Wochenarbeitszeit eingeschränkt. Die Planstellen im Krankenhaus mussten aufgestockt werden. Sichere Anstellungen im Spital werden bevorzugt. Werden die Kolleginnen durch die Arbeit in den Akutambulanzen abgeschreckt?

\section{Ärztinnen im Vormarsch}

Die gesamte Medizin wird weiblich. In der Kinder- und Jugendheilkunde ist dieser Trend verstärkt spürbar. Kassenstellen mit hohen Patientenfrequenzen sind allerdings für viele Kolleginnen nicht das Ziel ihrer Lebensplanung. Modelle für Gemeinschaftspraxen sind rar und noch nicht ausgereift. Krankenkassen fürchten Mehrkosten und bremsen.

Als Pädiater treten wir meist eher bescheiden auf. Als verantwortungsbewusste Ärzte müssen wir auch gesundheitspolitische Aspekte in den Fokus rücken. Bei einer Jahrestagung kann mehr Aufmerksamkeit erreicht werden als sonst. Wir wollen/müssen vermehrt wahrgenommen werden! $\mathrm{Zu}$ den Eröffnungsveranstaltungen, bei denen auch Vertreter der Politik anwesend sind, kommen meist sehr wenige Kongressteilnehmer. Das macht kein gutes Bild und stärkt nicht unsere Position.

\section{Gesundheitspolitik für Kinder und Jugendliche}

Wir haben Vertreter des Ministeriums und des Hauptverbands in eine Plenarsitzung eingeladen, um mit ihnen unsere Probleme zu diskutieren und an Lösungsvorschlägen zu arbeiten. Unsere Präsi- dentin, Univ.-Prof. Dr. Daniela Karall, und Univ.-Prof. Dr. Reinhard Kerbl werden durch die Sitzung führen. Eine solche Runde braucht einen vollen Saal und eine lebhafte Diskussion! Nehmen Sie sich Zeit, bringen Sie drängende Fragen, diskutieren Sie mit.

Am Samstag haben wir gemeinsam mit Kollegen Sedlak und Voitl Kollegen aus Deutschland der Schweiz und Frankreich eingeladen. Sie werden die Situation der Pädiatrie in der freien Praxis in ihren Ländern vorstellen. Wir erhoffen uns Anregungen für Verhandlungen, um eine bessere Versorgung in Österreich erzielen zu können.

\section{Aus der Praxis für die Tagung}

Bei der diesjährigen Jahrestagung haben wir erstmalig einen durchgehenden Track vom Donnerstag bis Samstag vorgesehen, der von Kollegen aus der freien Praxis geplant und moderiert wird. Damit soll sichergestellt werden, dass wir nicht an den Fortbildungswünschen der Kollegen vorbeiplanen.

\section{Seltene Erkrankungen}

Eine weitere Plenarsitzung ist der Behandlung seltener Erkrankungen gewidmet. Aufgrund technischer Entwicklungen wird es immer einfacher, Enzyme, Antikörper und genmodulierende Substanzen zu produzieren. Die Kosten dieser Therapien belasten bzw. bedrohen unsere Gesundheitsbudgets. Jahreskosten von $500.000 €$ und mehr pro Patient sind keine Seltenheit. Es ist unsere Aufgabe, für diese neuen Behandlungsmöglichkeiten einzutreten. Es sind aber schon so viele neue Substanzen in der Pipeline, dass die Finanzierbarkeit an ihre 
Grenzen stoßen wird. Die Entscheidung Therapie - Ja oder Nein darf nicht von Ökonomen gefällt werden. Enzymersatztherapien werden zum Teil auch in den Praxen verabreicht. Eine einheitliche Finanzierung unabhängig von den derzeitigen Gesundheitsbudgets ist zu fordern, um die Versorgung sicherzustellen.

\section{Über 100 Abstracts}

Die Tagung soll den jungen Kolleginnen in der Ausbildung die Möglichkeit geben, ihre Forschungsergebnisse vorzustellen. Erfreulicher Weise wurden über $100 \mathrm{Ab}$ stracts eingereicht.

\section{Tertiär- und Quartärversorgung}

Zur Kinderheilkunde gehört auch eine hochspezialisierte Tertiär- und Quartärversorgung. Arbeitsgruppen berichten von Entwicklungen in ihrem Bereich. Mittlerweile haben wir so viele Subspezialitäten, dass wir nicht für alle Sitzungen anbieten können. Falls Sie heuer nicht berücksichtigt werden konnten: Bitte im nächsten Jahr darauf hinweisen! Dieses Spezialwissen wird unseres Erachtens noch viel zu wenig in den Ordinationen angeboten. Sicher auch ein Thema der Finanzierung, das bei der Plenarsitzung angesprochen werden kann.

\section{Fokus Genetik}

Die pädiatrische wissenschaftliche Literatur wird von der Genetik bestimmt. Man hat manchmal den Eindruck, dass ohne Genetik nichts mehr geht. Dabei wird übersehen, dass über $99 \%$ aller pädiatrischen Erkrankungsfälle ohne jegliche genetische Untersuchung auskommen. In dem Spannungsfeld - was ist diagnostisch sinnvoll - was hilft - was belastet - arbeiten wir. Die Zahl der bekannten Gendefekte bewegt sich auf 8000 zu. Wir haben für viele bis dato unklare Krankheitsbilder Erklärungen bekommen. Die Kosten für Panels sinken und werden vermehrt eingesetzt. Seltene Erkrankungen werden wieder zur Entwicklung von teuren „orphan drugs“ führen. Damit schließt sich der Kreis. Wir haben die Planer der einzelnen Sitzungen gebeten, in einem Vortrag auf genetische
Entwicklungen in ihrem Fachbereich einzugehen.

\section{Liebe Leserinnen und Leser,}

wir hoffen, dass Ihr Interesse an der Jahrestagung geweckt wurde und Sie nach Linz kommen. Nochmals möchte ich darauf hinweisen, dass wir dann eher gehört werden, wenn wir uns in großer Zahl hinter unsere Forderungen stellen.

Wir freuen uns auf Ihr Kommen

Ihr

Klaus Schmitt

Tagungspräsident 2018

Ariane Biebl

Tagungssekretärin 2018

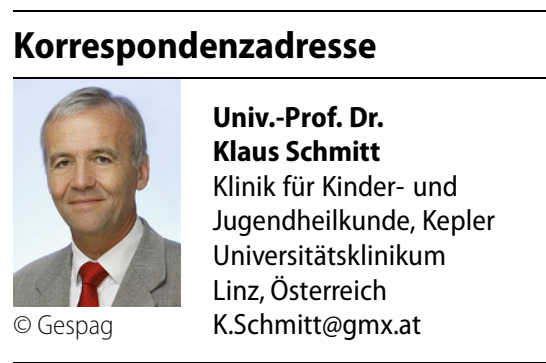

Interessenkonflikt. K. Schmitt gibt an, dass kein Interessenkonflikt besteht. 
Hier steht eine Anzeige.

\section{曾 Springer}

